\title{
Laboreal
}

Volume $17 \mathrm{~N}^{\circ} 1$ | 2021

Trabalhar hoje: mudanças, permanências, estratégias, reinvenções

\section{Subjetividad y trabajo: entre el mal-estar y el bien- estar - Jorge Juan Román Hernández, Dominique Lhuilier, José Newton García de Araújo y Andrea Pujol}

Subjetividade e trabalho: entre mal-estar e bem-estar - Jorge Juan Román Hernández, Dominique Lhuilier, José Newton García de Araújo e Andrea Pujol Subjectivité et travail: entre mal-être et bien-être - Jorge Juan Román Hernández, Dominique Lhuilier, José Newton García de Araújo et Andrea Pujol Subjectivity and work: between ill-being and well-being - Jorge Juan Román Hernández, Dominique Lhuilier, José Newton García de Araújo and Andrea Pujol

\section{Gilles Amado}

Traductor. Andrea Pujol (pujol.andrea@gmail.com)

\section{OpenEdition}

\section{Journals}

\section{Edición electrónica}

URL: https://journals.openedition.org/laboreal/17515

DOI: $10.4000 /$ laboreal. 17515

ISSN: 1646-5237

Editor

Universidade do Porto

Referencia electrónica

Gilles Amado, «Subjetividad y trabajo: entre el mal-estar y el bien-estar - Jorge Juan Román Hernández, Dominique Lhuilier, José Newton García de Araújo y Andrea Pujol», Laboreal [En línea], Volume $17 \mathrm{~N}^{0} 1$ | 2021, Publicado el 18 junio 2021, consultado el 20 junio 2021. URL: http:// journals.openedition.org/laboreal/17515 ; DOI: https://doi.org/10.4000/laboreal.17515

Este documento fue generado automáticamente el 20 junio 2021.

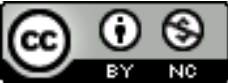

Laboreal está licenciado com uma Licença Creative Commons - Atribuição-NãoComercial 4.0 Internacional. 


\section{Subjetividad y trabajo: entre el mal- estar y el bien-estar - Jorge Juan Román Hernández, Dominique Lhuilier, José Newton García de Araújo y Andrea Pujol}

Subjetividade e trabalho: entre mal-estar e bem-estar - Jorge Juan Román Hernández, Dominique Lhuilier, José Newton García de Araújo e Andrea Pujol Subjectivité et travail: entre mal-être et bien-être - Jorge Juan Román Hernández, Dominique Lhuilier, José Newton García de Araújo et Andrea Pujol Subjectivity and work: between ill-being and well-being - Jorge Juan Román Hernández, Dominique Lhuilier, José Newton García de Araújo and Andrea Pujol Gilles Amado

Tradución : Andrea Pujol (pujol.andrea@gmail.com)

\section{NOTA DEL EDITOR}

Manuscrito recibido en: $16 / 11 / 2020$

Aceptado tras peritaje en: 09/04/2021

\section{Subjetividad y trabajo en tres idiomas}

Esta obra es particularmente interesante a raíz de su originalidad. Desarrolla en tres idiomas (francés, español, portugués) un número importante de comunicaciones presentadas por investigadores cubanos, latinoamericanos y francófonos (Francia, Quebec, Suiza) durante el simposio internacional realizado en octubre de 2018 en La 
Habana (Cuba) sobre el tema "Subjetividad y trabajo". Este evento reunió alrededor de 450 participantes que representan aproximaciones disciplinarias diversas (ergonomía, psicología del trabajo, medicina del trabajo, sociología, economía, derecho, filosofía, psicoanálisis). Bajo la premisa de una humanización del trabajo, en particular a través de la exploración de interrogantes en torno a la salud laboral, el encuentro permitió la elaboración de producciones científicas y a su vez la presentación de acciones de prevención implementadas en diferentes entornos de trabajo.

2 Para dar cuenta de dicha tarea, se han seleccionado cinco temas que encabezan los capítulos: transformación del trabajo y subjetividad; tiempo, actividad y subjetividad; género, salud y trabajo; trabajo precario, trabajo informal, trabajo invisible; investigación e investigación-acción.

\section{Un breve resumen de cada una de las contribuciones}

El primer texto de este libro (del psicólogo cubano, anfitrión y coorganizador del simposio Jorge Juan Román Hernández) denuncia el engaño que representa la exigencia de compromiso por parte de los trabajadores, una actitud valorada por los empresarios y un concepto querido por los especialistas del comportamiento organizacional y los teóricos norteamericanos de la gestión. El autor muestra que, si bien el compromiso puede promover efectos positivos en la salud ocupacional, también puede conducir a un mayor desgaste y sufrimiento físico y psicológico. Además, como estrategia de gestión, puede ser fuente de un proceso de alienación.

Danièle Linhart, cuyos escritos en sociología del trabajo tienen autoridad en Francia, se orienta en la misma dirección al señalar que esta gestión "vertiginosa" que reclama la autonomía de los empleados, en realidad la obstaculiza con todo tipo de dispositivos, servicios y herramientas impuestas en el marco de una relación de subordinación que en realidad favorece a la individualización y la competencia, fuentes de tensiones sociales e intrapsíquicas.

5 El presentismo - presencia en el trabajo a pesar de problemas de salud no expresados es uno de los fenómenos estudiados por la psiquiatra Edith Seligmann Silva sobre la base de una extensa investigación internacional. Indaga en sus raíces sociales, así como sus manifestaciones dentro de diversas organizaciones y su expansión, particularmente en Brasil, dando cuenta además de los desafíos éticos que plantea el tema.

6 A continuación, João Batista Ferreira, psicólogo, explora varios dispositivos de captura de la vitalidad orientados a la productividad propios del mundo neoliberal, así como las formas de resistencia que se oponen a esta modalidad de explotación. La investigación se basa en cinco estudios que utilizan el método de mapeo de formas de vida realizados con contratistas, electricistas, profesores y psicólogos.

7 Marc-Eric Bobillier-Chaumon, psicólogo del trabajo y especialista en el impacto de las tecnologías en el mundo del trabajo, muestra cómo se están implementando actualmente las nuevas generaciones de tecnologías, lo que induce tanto limitaciones como nuevos recursos que probablemente cambien los oficios y las habilidades. El compromiso subjetivo de los profesionales en estos entornos no está exento de riesgos si las herramientas desarrolladas siguen esencialmente una lógica tecnocéntrica, en 
detrimento de un enfoque más antropocéntrico basado en las capacidades y la inteligencia práctica.

El texto propuesto por Selma Lancman y Seiji Uchida toma como objeto las políticas públicas de salud ocupacional en Brasil. Su intervención, enmarcada en la psicodinámica del trabajo, se llevó a cabo con gerentes de los centros de salud ocupacional de Sao Paulo y permitió resaltar las distintas dimensiones que operan en la construcción de la intersectorialidad y en los servicios de asistencia a los trabajadores.

9 Dos estudios realizados por el Instituto Nacional de Salud de los Trabajadores (Cuba) a partir de bases de datos y análisis estadísticos en el sector de la construcción, analizaron los recursos psicológicos de los trabajadores (considerados como un factor clave de la salud) en relación con la capacidad de trabajo. De acuerdo con las conclusiones de Georgina López Pulmar, Felix Amador Romero y Roselia Bustamante Rojas, sería conveniente otorgar una importancia tangible a estos recursos psicológicos individuales cuando se desee diseñar estrategias de intervención y formación en la perspectiva de la salud ocupacional.

10 A continuación, la psicóloga argentina Andrea Pujol presenta un conjunto de reflexiones a partir de una investigación acción sobre el sufrimiento vivido por los docentes universitarios argentinos ante las transformaciones institucionales, en particular por la "gestionarización" de la vida académica. Esto conduce a una sobrecarga de tareas administrativas y conflictos éticos que se articulan con la disminución de la participación y la restricción de la autonomía profesional. En tal sentido, se sugiere la creación de espacios de deliberación entre pares, capaces de contribuir a repensar el trabajo y afrontar el malestar.

11 En el seno de la universidad pública colombiana es donde Daniel Ernesto Gómez Giraldo, Lina Marcela Gil Congote y Johnny Orejuela Gómez han estudiado -desde una aproximación fenomenológica- la experiencia de diez investigadores respecto del reconocimiento del trabajo. Los autores muestran que la actividad de enseñanza, la socialización en la comunidad científica y el impacto de su trabajo en el medio son los elementos clave en torno a los cuales se produce el reconocimiento, y en contrapartida, correría peligro cuando está ligado aspectos institucionales.

Dos ergónomas, investigadoras de la cuestión del tiempo en el trabajo, Corinne Gaudart y Sophie Prunier-Poulmaire, desde un enfoque holístico del tiempo de trabajo y basándose en historias de situaciones concretas cercanas al trabajo y su organización, defienden la idea de que el tiempo cuantitativo (horas y duraciones) no puede separarse del tiempo cualitativo (lo que hacemos allí), ni soslayar sus numerosos efectos deletéreos sobre la salud. Se trata entonces de una articulación bien pensada de estos dos aspectos que permitiría a los trabajadores apropiarse del tiempo preservando su salud.

13 El estudio de los efectos de los dispositivos de inserción laboral para sujetos que egresan de cárceles u hospitales psiquiátricos en Argentina, permitió a Silvana Melisa Herranz señalar ciertos elementos paradójicos de las exigencias institucionales: por una parte, el mundo profesional que privilegia la posición de un sujeto "autónomo" y "emprendedor" y por otra ¿cómo acceder a esa autogestión en las instituciones de "rehabilitación", que prescriben ante todo dependencia y sumisión? Se proponen aquí las discusiones resultantes de un análisis crítico de trabajos de investigación sobre este tema. 
Las investigaciones sobre las relaciones entre género y trabajo se desarrollan hoy en todo el mundo. Entre estos estudios, el de María de Lourdes Marrero Santos y sus colegas cubanos es particularmente original. Estudia el impacto de ciertos factores de riesgo en el trabajo en la concepción de bebés en una cohorte de 521 trabajadoras embarazadas pertenecientes a diversas profesiones. Junto a los riesgos clásicos relacionados con el entorno físico y disergonómico, esta investigación arroja luz sobre el estrés en el trabajo como riesgo "perinatal", cuya consideración se recomienda a la hora de contratar trabajadores.

En Chile, Elisa Ansoleaga y Alba Barrera mostraron la prevalencia de la violencia psicológica y sexual contra las mujeres en diferentes sectores de la economía y considerando tres grandes áreas metropolitanas (Santiago, Concepción, Valparaíso). Su metodología se basó en un estudio cuantitativo transversal representativo de la población asalariada.

6 Finalmente, también en el tema de género, Claudine Schalck, partera e investigadora en psicología ocupacional, exploró la violencia obstétrica, es decir, el maltrato que sufren las mujeres durante el nacimiento de su hijo. Perjudicial para las mujeres, esta violencia también es dañina para los cuidadores, a menudo obligados a hacer "trabajo sucio". De ahí la necesidad de construir una auténtica asociación entre parturientas y cuidadores para que se pueda valorar el reconocimiento y la autoestima.

7 Otro aspecto del trabajo es que puede ser precario, informal o invisible. Este es el tema del cuarto capítulo de este libro. Un equipo de investigadores brasileño - Jorge Tarcísio da Rocha Falcão, Cleverson Pereira de Almeida, Maria Regina Greggio y José Newton Garcia de Araújo - discuten tres casos de actividades precarias en diferentes contextos, los de un vigilador nocturno, un anatomopatólogo y un maestro "polifacético". Desde el abordaje de la clínica de la actividad y la noción de "trabajo sucio", los autores afirman que la precariedad está ligada a la disminución del trabajo colectivo, íntimamente vinculada a manifestaciones de sufrimiento en el trabajo.

El capítulo final está dedicado a la transformación del trabajo a través de la investigación y de la investigación-acción.

Se abre con la contribución de Ludmila de Vasconcelos, Fernanda Tarabal, Admardo Gomes Junior y Jacques Rhéaume, quienes presentan diversas aproximaciones teórico-clínicas al trabajo susceptibles de clarificar temas de salud y las modalidades de intervención que facilitan la emergencia de los aspectos vitales y arrojan luz sobre sus desafíos. Así, este equipo multicultural y multidisciplinario detalla los posibles vínculos entre la ergología y el psicoanálisis, la psicodinámica del trabajo, la investigaciónacción, el enfoque socioclínico, la psicosociología y las historias de vida.

20 Enio Rodrigues da Silva, Maristela Pereira y Vanessa Andrade de Barros, psicólogos brasileños, arrojan luz sobre el concepto de gesto profesional considerada una categoría analítica centrada en la actividad. Desde este punto de vista se detallan dos ejemplos de intervención en el sector hospitalario, apoyados en referencias a la ergología y la psicosociología del trabajo.

21 El texto propuesto por Fernanda Spanier Amador y Maria Elizabeth Barros de Barros analiza los movimientos (continuidad / discontinuidad) que se dan entre la "normatividad vital" y la "normatividad social", con referencia a la obra de G. Canguilhem e Y. Schwartz. Estas reflexiones se basan en datos recolectados durante las 
intervenciones realizadas en el campo de las políticas públicas brasileñas, particularmente en el ámbito de la educación social.

Desde la perspectiva de la clínica de la actividad, Luciana Gomes Albuquerque y Claudia Osorio da Silva presentan una intervención clínica en un centro de atención psicosocial brasileño y resaltan los beneficios del diálogo sobre el trabajo para aumentar su calidad.

Ante el sufrimiento en el trabajo de los docentes quebequenses debido a una problemática en la organización del trabajo, Simon Viviers, Marie-France Maranda, Nathalie Morel y Isabelle Ruelland han desarrollado, con una federación sindical de docentes, un sistema de intervención destinado a restaurar el poder de actuar y ofrecer así un destino "político" al sufrimiento.

En Francia, Malika Litim y Julie Cochin llevaron a cabo una investigación-acción dentro de una comunidad local sobre el tema de la reclasificación del personal. El dispositivo original, tomando en cuenta aspectos tanto preventivos como curativos, condujo a la implementación de una serie de acciones y a una evolución del sistema de gobernanza.

Finalmente, Dominique Lhuilier, coorganizador de la conferencia de La Habana, concluye este libro con un comentario original en el campo de la psicología del trabajo: el trabajo de salud. Se trata en este caso de las estrategias que implementan los empleados cuya salud se ve comprometida para construir mediaciones entre las exigencias del medio ambiente y la preservación de la salud. Por tanto, el autor muestra que las compensaciones y las estrategias tienen un doble objetivo, adaptativo y evolutivo.

\section{Al final de la lectura del libro}

A pesar de la diversidad de referentes teóricos, metodológicos y culturales, quedamos impactados por la proximidad de los hallazgos y reflexiones sobre salud ocupacional.

El deterioro de las condiciones de trabajo y la hegemonía de la gestión parecen traspasar fronteras y ofrecer así, por fortuna (?) terrenos a elección a los investigadores y profesionales para la observación y análisis de estos fenómenos, pero sobre todo para intentar llevar a cabo acciones orientadas a rehabilitar una cierta humanidad en el mundo del trabajo.

No hay duda de que investigadores y profesionales de todos los ámbitos encontrarán material valioso en este trabajo. 


\section{AUTORES}

\section{GILLES AMADO}

École des hautes études commerciales (HEC) ; Centre de Recherche sur le Travail et Développement (CRTD) ; Conservatoire National des Arts et Métiers (CNAM), 41, Rue Gay Lussac 75005 Paris, France. amado@hec.fr 